\title{
O papel da Consciência Fonológica na leitura contextual medida pelo teste de Cloze
}

\author{
Márcia Maria Peruzzi Elia da Mota \\ Universidade Federal de Juiz de Fora \\ Acácia Aparecida Angeli dos Santos \\ Universidade São Francisco - SP
}

\begin{abstract}
Resumo
Estudos que investigam o papel das habilidades metalingüísticas na leitura demonstram que a habilidade de refletir sobre os sons da fala, a consciência fonológica, contribui para leitura de palavras isoladas e compreensão de texto. Considerando a importância do tema, esta pesquisa foi realizada com o objetivo de explorar as relações entre a consciência fonológica e a compreensão em leitura. Participaram 19 crianças da $1^{\underline{a}}$ série e 23 da $2^{-}$série, cujas médias de idade foram respectivamente, 87,5 meses $(D P=3,93)$ e 98,3 meses $(D P=4,58)$. Os resultados mostraram que os escores nas tarefas de consciência fonológica se associaram aos escores do teste de Cloze, utilizado como medida da compreensão em leitura. Os resultados são discutidos à luz das teorias sobre aquisição da leitura e sugere-se a realização de novas pesquisas.
\end{abstract}

Palavras-chave: consciência metalingüística; avaliação; teste de Cloze; leitura

\begin{abstract}
The role of phonological awareness in contextual reading measured by the Cloze task.

Studies that investigate the role of metalinguistic abilities in reading show that the ability to reflect upon word's sound, phonological awareness, contributes to reading single words and reading comprehension. Considering the importance of this theme, this research was carried out with the objective of exploring the crelationship between phonological awareness and reading comprehension. Nineteen children from $1^{\text {st }}$ grade and 23 from $2^{\text {nd }}$ grade, whose mean ages were 87,5 months $(\mathrm{SD}=3,93)$ and 98,3 months $(\mathrm{SD}=$ $4,58)$ participated in the study. The results show that the scores on the phonological awareness tasks were associated to the Cloze's score, used as a reading comprehension measure. The results are discussed on the light of the theories of reading acquisition.
\end{abstract}

Keywords: metalinguistic awaraness; assessment; Cloze's test; reading.

$\mathrm{T}$ eóricos que estudam o ensino da leitura escrita têm se dividido em dois grupos: de um lado, aqueles que defendem um ensino mais diretivo da alfabetização, com a volta do ensino das correspondências entre letra e som (Capovilla \& Capovilla, 1997, 2000), e, do outro lado, encontram-se aqueles que defendem uma menor ênfase na decodificação e a utilização de informações contextuais no ensino da leitura e da escrita (Goodman, 1982a, 1982b; Jolibert, 1984; Smith, 1971).

Os estudiosos que defendem um ensino menos diretivo da escrita encontram subsídios teóricos e metodológicos nos trabalhos de Goodman (1982a, 1982b). Segundo esse autor, a leitura pode ser vista como um jogo de habilidades psicolingüísticas. A criança deveria ser encorajada a, através do seu conhecimento da língua materna, utilizar pistas sintáticosemânticas para adivinhar as palavras do texto.

A visão de Goodman (1967) dominou as práticas de leitura até a década de 80 , quando começou a ser posta em xeque. Evidências empíricas têm demonstrado que o efeito facilitador do contexto observado por Goodman desaparece quando controles como contrabalanceamento de condições são aplicados. Nicholson (1993) chamou atenção para o fato de que, no estudo original de Goodman (1965), as palavras lidas em isolamento foram apresentadas antes das lidas no contexto. Alterando a ordem de apresentação das condições, o autor mostrou que se elimina o fator facilitador do contexto.

Além disso, alguns autores começaram a sugerir que as crianças podem utilizar pistas contextuais associadas a pistas grafo-fonêmicas para aprender a ler (Rego, 1991; Tunmer, Herriman, \& Nesdale, 1988). Dessa forma o contexto atuaria como suporte quando a criança só conseguisse decodificar parcialmente as palavras no texto.

O outro grupo de teóricos a que nos referimos defende que a decodificação deve ter um papel central na leitura e escrita. 
Essa visão é apoiada pelos trabalhos que estudam o papel das habilidades metalingüísticas na alfabetização, especificamente as pesquisas sobre a consciência fonológica.

Nos últimos 30 anos houve um aumento expressivo do número de estudos que investigaram a relação entre a consciência metalingüística e a alfabetização (Bowey, 2005; Bradley \& Bryant, 1983; Gombert, 1992; Plaza \& Cohen, 2003, 2004). Dentre as habilidades metalingüísticas, a mais estudada é a consciência fonológica, que pode ser definida como a habilidade de refletir sobre os sons que compõem a fala (Cardoso-Martins, 1995).

Vários autores têm demonstrado que a consciência fonológica ajuda na alfabetização, e que o treinamento dessa habilidade freqüentemente tem sido utilizado visando à remediação dos problemas de leitura (Bradly \& Bryant, 1985; Cuninghan, 1990; Goswami \& Bryant, 1990). No Brasil, os trabalhos de Barrera e Maluf (2003), Capovilla e Capovilla (2000), Cardoso-Martins (1995), Guimarães (2003), Petsun (2005), Santos (1996) e Zucoloto e Sisto (2002), só para citar alguns, confirmam a importância do tema para aquisição da língua escrita no português brasileiro.

O princípio que norteia esses trabalhos é o de que a criança que é capaz de refletir sobre os sons da fala teria mais facilidade de associar esses sons às letras, adquirindo o princípio alfabético. Essa hipótese foi testada por meio de estudos longitudinais que mostraram que a habilidade de refletir sobre os sons da fala em crianças não leitoras prediz o sucesso na leitura depois do início da alfabetização formal (Bradly \& Bryant, 1983; Rego \& Bryant, 1993).

Embora a consciência fonológica geralmente esteja associada à decodificação de palavras, poucos estudos têm estudado como essa habilidade contribui para leitura contextual ou compreensão de leitura (Rego, 1995; Rego \& Bryant, 1993; Tunmer, 1990). Um dos primeiros trabalhos de pesquisa que explorou a função das habilidades metalingüísticas na leitura contextual foi o de Tunmer, Herriman e Nesdale (1988). Esses autores realizaram um estudo longitudinal em que verificaram o papel de diferentes tipos de habilidades metalingüísticas no desempenho da leitura com diferentes tipos de estímulo (leitura contextual, leitura de palavras isoladas). Observaram que a consciência fonológica relacionava-se especificamente com a decodificação na leitura enquanto que a consciência sintática, habilidade de refletir sobre os aspectos sintáticos das sentenças, contribuía tanto para a compreensão da leitura como para a decodificação.

Estudos brasileiros têm enfatizado a importância da continuidade dos estudos sobre as implicações do desenvolvimento da consciência fonológica para a aprendizagem da leitura, especialmente no que se refere à compreensão. $\mathrm{O}$ estudo de Guimarães (2003) revelou que o desempenho em tarefas de consciência fonológica antes do aprendizado da leitura tem relação direta com o desempenho posterior nessa habilidade. Outros autores têm salientado que a consciência fonológica e a aprendizagem da leitura ocorrem em interação, uma vez que o sistema alfabético de escrita, utilizado na língua portuguesa, associa um componente auditivo fonêmico a um componente visual gráfico, favorecendo o conhecimento do princípio alfabético e, consequentemente, as regras de correspondência entre grafemas e fonemas (Barrera, 2003; Barrera \& Maluf, 2003, Guimarães, 2005).

O trabalho de Pestun (2005) teve o propósito de verificar se crianças que não possuíam estimulação prévia pela pré-escola apresentavam consciência fonológica ao ingressarem no ensino formal e se a presença dessa habilidade favorecia a aquisição da leitura e escrita. Foram investigadas 167 crianças de ambos os sexos, com idade média de 5 anos e 8 meses, tendo sido a coleta de dados realizada em etapas distintas, sendo uma delas para avaliação da consciência fonológica. Em outra etapa, as crianças foram avaliadas em leitura oral e escrita com ditado de palavras e de pseudopalavras. Na mesma direção dos estudos anteriores mencionados, a autora detectou a existência de correlação positiva entre consciência fonológica e o desempenho em leitura e em escrita.

Anteriormente, Tunmer, Herriman e Nesdale (1988) já haviam hipotetizado que crianças utilizam pistas grafofonêmicas associadas a pistas contextuais para ler palavras que não conhecem quando elas são apresentadas no contexto. Dessa forma a habilidade de refletir sobre as regras sintático-semânticas no contexto acaba colaborando com a decodificação. Por essa razão, correlações positivas e significativas são encontradas entre a consciência sintática e a decodificação.

No estudo longitudinal realizado por Rego e Bryant (1993) com crianças inglesas essa hipótese foi testada. Os autores constataram que havia uma relação específica entre desempenho inicial em tarefas de consciência sintática e a leitura no contexto e de palavras isoladas, ao passo que a consciência fonológica contribuiu apenas para decodificação. Assim sendo, concluíram que a consciência fonológica e a consciência sintática facilitam a leitura de formas diferentes. A consciência fonológica teria um impacto específico sobre a decodificação e a consciência sintática sobre a leitura contextual e a decodificação. A consciência sintática auxiliaria na decodificação porque as pistas contextuais ajudariam a criança a decodificar os "pedacinhos" das palavras que não consegue ler. Esse conhecimento seria generalizado para outras palavras auxiliando a aquisição do princípio alfabético.

Diferentes ortografias podem requerer diferentes estratégias de leitura e escrita. Rego (1995) questionou se esses resultados poderiam ocorrer como conseqüência das características da ortografia inglesa. Estava interessada em saber se poderiam ser generalizados para o português, uma língua com correspondência letra e som mais regular que o inglês. Em um estudo longitudinal com crianças falantes do português, a autora verificou que a consciência fonológica também não contribuiu para a leitura contextual nessa ortografia. Apenas os escores de consciência sintática produziram um resultado significativo para leitura.

Já apresentamos o argumento de Tunmer, Herriman e Nesdale (1988) de que a decodificação parcial das palavras associadas a pistas contextuais ajuda a criança a apreender o princípio alfabético. É nossa opinião que nesse caso algum tipo de processamento fonológico deveria estar presente na leitura contextual. Além disso, tem-se argumentado que a automatização da decodificação acelera o processo de reconhecimento das palavras no texto que, por sua vez, libera espaço da memória para armazenamento das informações contextuais contidas nele, facilitando o processo de compreensão (Stanovich, 1980). 
Levando em conta esses dois argumentos era de se esperar que tanto a consciência fonológica quanto a sintática contribuísse para leitura contextual. Nesse sentido, seria esperado que a consciência sintática ajudasse na utilização de regras contextuais e a consciência fonológica auxiliasse na aquisição do princípio alfabético, permitindo que partes decodificadas das palavras servissem de pista para escolha de palavras que poderiam ser completadas a partir do contexto.

Contudo, observa-se que os estudos realizados no português do Brasil têm demonstrado que a consciência fonológica está associada à leitura de palavras isoladas e à decodificação. Mas, poucos estudos foram realizados para investigar a relação entre a consciência fonológica e a leitura contextual. A partir da reanálise dos dados de Mota et al. (2009) pretendemos responder a essa lacuna na literatura. Escolhemos o teste de Cloze como medida de leitura contextual, visto que sua forma de elaboração permite que se analise o quanto o leitor se apóia nas pistas do contexto.

Vale ressaltar que o teste de Cloze foi escolhido porque ele pressupõe que a compreensão de leitura é resultante de processos cognitivos subjacentes que incluem, não apenas a decodificação dos símbolos e a percepção de pistas do próprio texto, como também a recuperação de conhecimentos prévios contidos na memória. Em razão disso o teste de Cloze tem sido uma das medidas mais utilizadas para avaliação da compreensão da leitura, havendo pesquisas que demonstram evidências de sua validade e precisão (Greene Jr, 2001; Hussein, 1999; Oliveira e Santos (2005); Santos, Primi, Taxa \& Vendramini, 2002; Santos, 2005). Com base nas considerações apresentadas, o presente estudo foi proposto com o objetivo de explorar as possíveis relações entre o desempenho em tarefas de consciência fonológica e a compreensão em leitura, medida pelo teste de Cloze.

\section{Método}

\section{Participantes}

A amostra do estudo foi constituída por 42 crianças, sendo 19 alunas da $1^{\mathrm{a}}$ série e 23 da $2^{\mathrm{a}}$ série, ambas do ensino fundamental de escolas particulares, situadas na região urbana de Juiz de Fora. A média de idade das crianças de $1^{\underline{a}}$ série foi de 87,5 meses $(D P=3,93)$ e a da $2^{\underline{a}}$ série 98,3 meses $(D P=4,58)$. As crianças foram convidadas a participar por meio de uma carta convite. A participação no estudo dependeu da autorização do responsável através do Termo de Consentimento Livre e Esclarecido.

\section{Instrumentos}

Tarefas de consciência fonológica. Foi usada a versão brasileira adaptada por Cardoso-Martins (1997) do oddity test de Bradley e Bryant (1983), investigando a categorização de rima e aliteração. Nesta tarefa a criança deve decidir qual palavra não tem o mesmo som que outras em duas listas, sendo a primeira de rima (exemplo: 'morcego', 'panela' e 'janela') e a segunda de aliteração (ex.: 'selo', 'casa' e 'sopa'). São 12 pontos possíveis no total.

Cloze: Teste de Compreensão de Leitura. Esta tarefa consiste na organização de dois textos dos quais se suprimem alguns vocábulos, e se pede à criança que preencha os espaços com as palavras que melhor completarem o sentido dos textos. Para o presente estudo foram escolhidos dois textos, cujas propriedades psicométricas já tinham sido estabelecidas em estudo anterior no Brasil (Santos, 2005). Assim sendo, já havia sido identificado que ambos apresentavam evidências de validade (convergente e de critério) e também índices de consistência interna satisfatórios $(\alpha>0,70)$. O número de acertos possíveis para cada um dos textos é de 15 pontos, perfazendo o total de 30 pontos quando se considera o conjunto das duas histórias. Vale destacar que em estudo posterior, foi identificada a eficácia das provas também para crianças de $1^{\mathrm{a}}$ série do ensino fundamental (Suehiro, 2008).

Escala de Inteligência Wechsler para crianças-WISC III (Weschler, 1991). Apenas foram utilizados os subtestes de Vocabulário e Dígitos, recorrendo-se ao uso dos escores ponderados para as análises. Destaca-se que os dois subtestes da escala Wechsler escolhidos apresentam bons critérios de consistência interna medidos pelo critério de Guttman (coeficientes maiores que 0,60 ). A utilização de tais subtestes se deu visando ao controle da influência do desenvolvimento cognitivo na aquisição da leitura e escrita.

\section{Procedimento}

As crianças foram avaliadas individualmente em duas sessões de 20 a 30 minutos. Na primeira foram realizados os testes de consciência morfológica (não apresentados nesse artigo) e os testes de consciência fonológica. Na segunda sessão foram aplicados o Cloze - teste de compreensão de leitura - e os dois subtestes do WISC (Vocabulário e Dígitos).

$\mathrm{O}$ teste de consciência fonológica foi aplicado de forma oral e as orientações foram as mesmas descritas no item referente a esse instrumento.

No teste de Cloze solicitou-se às crianças completarem o texto da seguinte forma: "Eu vou dar para você uma estória em que está faltando alguns pedacinhos. Eu gostaria que você lesse a estória e depois completasse os pedacinhos que estão faltando. Mas, lembre-se, você só pode completar com uma palavrinha só".

Na segunda sessão foram aplicados os dois textos de Cloze, sendo pedido à criança que respondesse um de cada vez. Os textos foram sempre apresentados na mesma ordem. Solicitavase que lessem a história até o fim e depois voltassem ao início e preenchessem os espaços com as palavras que julgassem dar mais sentido ao texto. No mesmo dia os dois subtestes do WISC III (Vocabulário e Dígitos) foram aplicados segundo o procedimento padrão recomendado.

\section{Resultados}

Estudos sobre a consciência fonológica e sua relação com a leitura mostram que variáveis como memória e vocabulário podem afetar, como variáveis estranhas, a força das correlações entre essas variáveis (Bradley \& Bryant, 1983). Para verificar se havia uma associação entre a leitura contextual e a consciência fonológica, memória e vocabulário foi realizada a prova de correlação de Spearman. Para as tarefas de consciência 
fonológica e as duas versões do teste de Cloze, 1 ponto foi dado para cada resposta correta. A correção do Cloze foi verbatim, visto que não foram aceitos sinônimos ou palavras escritas de forma incorreta. A adoção dessa forma de correção evita que critérios subjetivos, dependentes da interpretação do experimentador, afetem a correção. A Tabela 1 mostra a média de acerto por série nas tarefas.

Os resultados mostram que os escores dos testes de Cloze correlacionaram-se de forma positiva e significativa com as duas medidas de consciência fonológica $[r=0,37 ; p<0,05]$

Tabela 1

Média e desvio padrão das medidas de leitura, consciência fonológica, vocabulário e inteligência

\begin{tabular}{cccccccc}
\hline Série & & Aliteração & Rima & Vocabulário & Dígito & Cloze 1 & Cloze 2 \\
\hline \multirow{2}{*}{$1^{\text {a }}$} & Média & 9,3 & 10,36 & 19,63 & 10,26 & 5,21 & 2,78 \\
& $(D P)$ & $(3,03)$ & $(1,49)$ & $(5,85)$ & $(2,20)$ & $(3,13)$ & $(2,20)$ \\
& & & & & & & \\
2 & & & & \\
& Média & 10,82 & 10,60 & 25,17 & 11,30 & 8,43 & 5,86 \\
& $(D P)$ & $(1,77)$ & $(2,33)$ & $(5,09)$ & $(1,91)$ & $(2,72)$ & $(1,96)$ \\
\hline \multirow{2}{*}{ Total } & Média & 9,07 & 9,92 & 20,67 & 10,01 & 6,97 & 4,47 \\
& $(D P)$ & $(3,04)$ & $(2,39)$ & $(6,11)$ & $(2,12)$ & $(3,30)$ & $(2,56)$ \\
\hline
\end{tabular}

para aliteração e o teste de Cloze 1 e $[r=0,37 ; p<0,05]$ para aliteração e o teste de Cloze 2; e $[r=0,40 ; p<0,01]$ para rima e o teste de Cloze 1 e $[r=0,51 ; p<0,01]$ para rima e o teste de Cloze 2.

A medida de vocabulário, ao contrário do esperado, não se correlacionou de forma positiva e significativa com os escores do Cloze. As medidas do Cloze apresentaram correlações positivas e significativas com a tarefa de memória de dígitos e com a idade. A tabela 2 mostra os coeficientes de correlação para as análises realizadas.

Como já mencionado acima, correlações podem ser afetadas por variáveis estranhas. É possível pensarmos, por exemplo, que as crianças mais velhas sejam melhores que as mais novas nas tarefas de dígitos, Cloze e consciência morfológica. As correlações entre essas variáveis podem ser decorrentes dos efeitos da idade. O mesmo tipo de raciocínio pode ser aplicado para as outras correlações significativas. Para investigar se a consciência fonológica contribuía para a leitura, independentemente do efeito das outras variáveis, três regressões lineares foram realizadas nos dados do teste de Cloze 2. O teste de Cloze 1 não apresentou uma distribuição adequada para esse tipo de análise.

Tabela 2

Coeficientes de correlação de Spearman entre as várias medidas

\begin{tabular}{|c|c|c|c|c|c|c|}
\hline & Cloze 2 & Idade & Vocabulário & Aliteração & Rima & Dígito \\
\hline Cloze 1 & $* * 0,72$ & $* * 0,47$ & 0,12 & $* 0,36$ & $* * 0,40$ & $* 0,31$ \\
\hline Cloze 2 & & $* * 0,50$ & 0,20 & $* 0,39$ & $* * 0,51$ & $* * 0,53$ \\
\hline Idade & & & $* 0,33$ & $* * 0,44$ & 0,27 & $* * 0,44$ \\
\hline Vocabulário & & & & 0,13 & $* 0,35$ & $* * 0,44$ \\
\hline Aliteração & & & & & $* * 0,52$ & $* * 0,47$ \\
\hline Rima & & & & & & $* * 0,54$ \\
\hline
\end{tabular}

As regressões realizadas foram de ordem fixa. A primeira variável a entrar na análise foi a idade, seguida dos escores de rima ou de aliteração. Os escores de rima continuaram a contribuir de forma significativa para o Cloze depois que a variância partilhada pela idade foi retirada da análise $[\beta=$ $0,30 ; t=2,3 ; p=0,026]$. Quando entramos o escore de dígito como segunda variável na equação os escores de rima deixam e contribuir de forma significativa para análise $[\beta=0,17 ; t=1,35$; $p=0,21]$.

Para aliteração não houve resultados significativos depois que a variância atribuída a idade foi controlada $(\beta=0,72 ; t=$ $0,48 ; p=0,84]$.

\section{Discussão}

Teorias sobre o desenvolvimento da leitura têm argumentado 
que diferentes tipos de leitura envolvem diferentes tipos de habilidade metalingüística. A consciência fonológica estaria associada à leitura de palavras isoladas, pois está ligada diretamente a aquisição do princípio alfabético, enquanto a leitura contextual estaria associada à consciência sintática, que envolve reflexão sobre informações sintático-semânticas envolvidas no contexto.

Evidência para essa hipótese foi apresentada por Rego (1995). Estudando crianças brasileiras a autora mostrou que tarefas de consciência fonológica realizadas pelas crianças aos 5 anos e 8 meses não prediziam a leitura contextual um ano depois. Porém, os escores no teste de consciência sintática predisseram a leitura dessas crianças.

Rego (1995) concluiu que em línguas alfabéticas, quando as crianças são ensinadas pelo método silábico como foi o caso das crianças estudadas por ela, a sensibilidade fonológica parece não ser relevante para a leitura contextual. Porém, se as crianças são ensinadas explicitamente a refletir sobre unidades de som das palavras, deveríamos esperar que elas generalizassem esse conhecimento para outras unidades de som que compõem a palavra. Uma explicação alternativa para os resultados de Rego é a de que um viés metodológico ocorreu. Em seu estudo, a autora utilizou apenas uma medida de consciência fonológica, uma medida de aliteração.

No presente trabalho a consciência fonológica foi medida por duas tarefas: uma de rima e outra de aliteração. Embora as duas tarefas tenham apresentado correlações positivas e significativas com a leitura contextual, a tarefa de rima manteve sua contribuição depois de controlarmos o efeito da idade, o que não ocorreu com a aliteração.

Importante destacar que embora as correlações tenham sido baixas entre o desempenho em leitura medido pelo Cloze e a tarefa de aliteração $(0,36$ a 0,39$)$, elas foram moderadas $(0,40$ a $0,51)$ quando estabelecidas com a tarefa de rima. Tais resultados são congruentes com vários dos estudos brasileiros aqui trazidos (Barrera, 2003; Barrera \& Maluf, 2003, Capovilla \& Capovilla, 2000; Cardoso-Martins, 1997; Pestun, 2005).

Quando a análise de regressão incluiu a memória de dígito como controle, a contribuição da rima deixou de ser significativa para leitura no Cloze. O teste de dígito usado nesse estudo é também um teste de processamento fonológico e contribuiu de forma significativa para leitura contextual. A inclusão do teste de dígito torna a análise de regressão muito conservadora. Portanto, consideramos que esses resultados dão suporte a idéias de que mesmo na leitura contextual algum tipo de processamento fonológico está presente.

Um último resultado que precisamos comentar não pode ser explicado no contexto da presente pesquisa. Era esperado que a pontuação no teste de vocabulário contribuísse de forma significativa para leitura contextual, tal como medida pelo Cloze. É importante lembrar que o pressuposto da técnica é que ela depende não apenas do uso das pistas imediatas fornecidas pelas palavras que antecedem e sucedem o traço a ser preenchido, mas também da memória, visto que necessita recuperar informações lá armazenadas (Santos, 2004; Oliveira \& Santos, 2005).

Dessa forma, nossos resultados oferecem evidências que confrontam a teoria de Goodman (1982a, 1982b, 1982c).
Sugerem que as práticas de ensino da leitura e da escrita devem envolver o ensino das regras de correspondência entre letra $\mathrm{e}$ som. Contudo, nossos resultados não excluem a importância do contexto no processo de formação de bons leitores. Questionamos a posição de que um método de alfabetização em particular venha a ser a panacéia para a solução dos problemas da alfabetização no Brasil.

O processamento da língua escrita é muito mais complexo do que a mera aquisição de um código, visto que engloba não só processos cognitivos, como também aspectos sociais e afetivos. As práticas de ensino da leitura e escrita deveriam considerar todas essas dimensões envolvidas. O desafio do educador é o de desenvolver práticas de ensino da leitura que visem a tornar o aprendiz proficiente na utilização do código escrito, ao mesmo tempo em que se trabalhem estas práticas em um contexto significativo, que insira a escrita na realidade social do aprendiz.

\section{Referências}

Barrera, S. D. (2003). Papel facilitador das habilidades metalingüísticas na aprendizagem da linguagem escrita. Em R. M. Maluf(Org.), Metalinguagem e aquisição da escrita: contribuições para a prática da alfabetização (pp. 19-63). São Paulo: Casa do Psicólogo.

Barrera, S., \& Maluf, M. R. (2003). Consciência metalingüística e alfabetização: um estudo com crianças da primeira série do ensino fundamental. Psicologia: Reflexão e Crítica, 16(3), 491-502.

Bowey, J. (2005). Grammatical sensitivity: its origins and potential contribution to early reading skill. Journal of Experimental Child Psychology, 90, 318343.

Bradley, L., \& Bryant, P. (1983). Categorizing sounds and learning to read - a causal connection. Nature, 301, 419-521.

Bradley, L., \& Bryant, P. (1985). Children's reading problem. Oxford: Basil Blackwells.

Capovilla, A., \& Capovilla, F. (1997). O desenvolvimento da consciência fonológica durante a alfabetização. Temas sobre o Desenvolvimento, $35(6), 15-21$.

Capovilla, A., \& Capovilla, F. (2000). Efeitos do treino de consciência fonológica em crianças com baixo nível sócio-econômico. Psicologia: Reflexão e Critica, 13(1), 7-24.

Cardoso-Martins, C. (1995). Consciência fonológica e alfabetização. Petrópolis: Vozes.

Cardoso-Martins, C. (1997). A sensibilidade à rima e ao fonema e a aquisição da leitura em crianças normais e individuos com a sindrome de Down. um estudo correlacional. Tese para obtenção de título de professor titular. Universidade Federal de Minas Gerais, Belo Horizonte.

Cuningham (1990). Implicit versus Explicit instruction in phonemic awareness. Journal of experimental child psychology, 50, 426-444.

Gombert J. E. (1992). Metalinguistic Development. Hertfordshire: Harverster Wheatsheaf.

Goodman, K. (1965). A linguistic study of cues and mescues in reading Elementary English, 42, 639-643.

Goodman, K. (1967). Reading: a psycholinguistic guessing game. Journal of the Reading Specialist, 6(4), 126-135.

Goodman, K. (1982a). Miscues windows on the reading processes. In F. V. Gollasch (Org.), Language and literacy: the selected writings of Kenneth S. Goodman (pp. 70-75). Boston: Routledge and Kegan Paul.

Goodman, K. (1982b). Miscues: "Windows on the reading process." In F. V. Gollasch (Org.), Language and literacy: the selected writings of Kenneth 
Goodman (pp.93-102). Boston: Routledge \& Kegan Paul.

Goodman, K. (1982c). "Analysis of oral reading miscues: Applied psycholinguistics". In F. V. Gollasch (Org.), Language and literacy: the selected writings of Kenneth Goodman (pp.123-134). Boston: Routledge $\&$ Kegan Paul.

Goswuami, U., \& Bryant P. (1990). Phonological skills and learning to read. London: Lawrence Erlbaun Associates.

Greene Jr., B. B. (2001). Testing reading comprehension of theoretical discourse with Cloze. Journal of Research in Reading, 24 , 82-98.

Guimarães, S. R. K. (2003). Dificuldades no desenvolvimento da lectoescrita: o papel das habilidades metalinguísticas. Psicologia: Teoria e Pesquisa, 19(1), 33-45.

Guimarães, S. R. K. (2005). Influência da variação lingüística e da consciência morfossintática no desempenho em leitura e escrita. Interação em Psicologia, 9(2), 261-271.

Hussein, C. L. (1999). Treino e generalização de leitura crítica e criativa: um estudo experimental com universitários. Estudos de Psicologia, 16(2), 16-27.

Jolibert, J. (1984). Formando crianças leitoras. Porto Alegre: Artes Médicas.

Mota, M. M. P. E., Lisboa, R., Dias J., Gontijo, R., Paiva, N., Mansur-Lisboa, S. F., Silva, D. A., \& Santos, A. A. A. (2009). Relação entre consciência morfológica e leitura contextual medida pelo teste de Cloze. Psicologia: Reflexão e Crítica, 22(2), 223-229.

Nicholson, T. (1993). The case against context. Em G. B., Thompson, W. Tunmer, \& T. Nicholson (Orgs.), Reading acquisition processes (pp. 91-103). Clevedon: Multilingual Matters.

Oliveira, K. L., \& Santos, A. A. A. (2005). Compreensão em leitura e avaliação da aprendizagem em universitários. Psicologia Reflexão e Crítica, 18(1), 118-124.

Pestun, M. S. V. (2005). Consciência fonológica no início da escolarização e o desempenho ulterior em leitura e escrita: estudo correlacional. Estudos de Psicologia (Natal), 10(3), 407-412.

Plaza, M., \& Cohen, H. (2003). The interaction between phonological processing, syntactic awareness, and naming speed in the reading and spelling performance of first-grade children. Brain and Cognition, 53, 257-292.

Plaza, M., \& Cohen, H. (2004). Predictive influence of phonological processing, morphological/syntactic skill, and naming speed on spelling performance.
Brain and Cognition, 55, 368-373.

Rego, L. (1991). The role of early linguistic awareness in children's reading and spelling. Tese de Doutorado, Universidade de Oxford, Oxford.

Rego, 1. (1995). Diferenças individuais na aprendizagem inicial da leitura: papel desempenhado por fatores metalingüísticos. Psicologia: Teoria e Pesquisa, $11(1), 51-60$.

Rego, L., \& Bryant, P. (1993). The connections between phonological, syntactic and semantic skills and children's reading and spelling. European Journal of Psychology, 3, 235-246.

Santos, A. A. A. (1996). A influência da consciência fonológica na aquisição da leitura e da escrita. In F. F. Sisto, G. C. Oliveira, L. D. T. Fini, M. T. C. C. Souza \& R. P. Brenelli (Orgs.), Atuação psicopedagógica e aprendizagem escolar (pp. 213-247). Petrópolis: Vozes.

Santos, A. A. A. (2004). O Cloze como técnica de diagnóstico e remediação da compreensão em leitura. Interação, 8(2), 217-226.

Santos, A. A. A. (2005). Evidências de validade de critério para o Teste de Cloze. Relatório Técnico. Programa de Pós-graduação em Psicologia, Universidade São Francisco, Itatiba, São Paulo.

Santos, A. A. A., Primi, R., Taxa, F., \& Vendramini, C. M. M. (2002). O teste de Cloze na avaliação da compreensão em leitura. Psicologia: Reflexão e Critica, 15(3), 549-560

Smith, F. (1971). Understanding Reading. New York: Holt, Rinehart \& Winston.

Stanovich, K. (1980). Towards an interactive compensatory model of individual differences in the development of reading fluency. Reading Research Quarterly, 1, 33-71.

Suehiro, A. C. S. (2008). Processos fonológicos e perceptuais e aprendizagem da leitura e escrita: instrumentos de avaliação. Tese de Doutorado, Universidade São Francisco, Itatiba, São Paulo.

Tunmer, W. (1990). The role of language prediction skills in beginning reading. New Zealand Journal of Educational Studies, 25(2), 95-112.

Tunmer, W., Herriman, M., \& Nesdale, A. (1988). Metalinguisic abilitiesand learning to read. Reading Research Quarterly, 23, 135-158.

Wechsler, D. (1991). WISC-III: Escala de inteligência Weschsler para crianças. São Paulo: Casa do Psicólogo, 1991.

Zucoloto, K. A., \& Sisto, F. F. (2002). Dificuldades de aprendizagem em escrita e compreensão em leitura. Interação em Psicologia, 6(2), 157-166.

Márcia Maria Peruzzi Elia da Mota, doutora em Psicologia pela Universidade de Oxford (Oxford University) - Inglaterra (OU), é professora titular do Programa de Pós-Graduação em Psicologia da Universidade Salgado de Oliveira (UNIVERSO) e professora adjunta da Universidade do Estado do Rio de Janeiro (UERJ). E-mail: mmotapsi@gmail.com Acácia Aparecida Angeli dos Santos, doutora em Psicologia e Desenvolvimento Humano pelo Instituto de Psicologia da Universidade de São Paulo (IP-USP), é professora titular do Programa de Pós-Graduação Stricto Sensu da Universidade São Francisco - USF. E-mail: acacia.santos@saofrancisco.edu.br 